\title{
Interaction between two nonpoint nanoparticles: Implementation to biology and medicine
}

\author{
V. Lozovski ${ }^{1,2}$, V. Lysenko ${ }^{2}$, V. Piatnytsia ${ }^{1}$, M. Spivak ${ }^{3}$ \\ ${ }^{1}$ Institute of High Technologies, Taras Shevchenko Kyiv National University, \\ 64, Volodymyrska str., 01601 Kyiv, Ukraine \\ ${ }^{2}$ V. Lashkaryov Institute of Semiconductor Physics, NAS of Ukraine, \\ 45, prospect Nauky, 03028 Kyiv, Ukraine \\ ${ }^{3}$ D. Zabolontyi Institute of Microbiology and Virology, NAS of Ukraine, \\ 154, Zabolotnyi str., 13143 Kyiv, Ukraine \\ Corresponding author e-mail: pyatnyx@gmail.com
}

\begin{abstract}
A new mechanism of interaction between two nanoparticles characterized by their dimensions and shape has been proposed. The mechanism is based on the local-field induced dipole momentum interaction with taking into account nonlinear polarizabilities of the particles. The dipole momentum induced in the particles has a fluctuating nature. The proposed mechanism of interparticle interaction leads to formation of both repulsive and attractive parts having the minimum at the distance between the particles close to their linear dimensions. The profile and strength of the potential depend both on dimensions and shapes of the particles as well as on their mutual orientation. Implementation of the discussed interaction conception to biology and medicine has been discussed.
\end{abstract}

Keywords: nanoparticle, local-field enhancement, vacuum field fluctuations, antiviral activity.

Manuscript received 27.08.12; revised version received 25.09.12; accepted for publication 17.10.12; published online 12.12.12.

\section{Introduction}

Modern technologies allows to fabricate nano-systems with preassigned properties [1-3], which can be used in various fields of human activity - physics [4], chemistry [5], biology [6] and medicine [7]. It should be noted that usage of results of nano-physics and nano-technologies in biology and medicine is a very important problem, solving of which promises in future revolution transformations in modern life sciences. Namely, the new methods of medical treatment of cancer - plasmonic photothermal therapy (PPTT) $[8,9]$, antivirus therapy [10-12], nanoparticles drug delivery $[13,14]$ improve the general state of play in modern medicine. The usage of new aspects of nanoplasmonics has led to development and fabrication of new types of different sensors $[15,16]$. When considering interaction between the nanoparticles, one should point out to at least three aspects of the problem. There are the dependences of interparticle interactions on the dimensions of the particles, on their shapes and relative positions. All these aspects lead to different influence on the interaction potential. It means that the use of different technological methods to fabricate nanoparticles can help us to obtain the interparticle potential with a preset profile.

The general relations characterizing the potential of interparticle interaction on the base of the scheme developed in the work [17] will be evaluated and analyzed in our work.

\section{Main equations}

Using the method of calculation of interparticle interaction between the non-point nanoparticles characterized both by linear and nonlinear polarizabilities developed in [17], we shall consider the general expressions describing the interaction potential. 


\subsection{Free energy of the system and ground state}

Let us consider two nanoparticles ('a' and 'b') characterized by any shapes and any dimensions. Let these particles are embedded into the medium of dielectric constant $\varepsilon$. The particles are characterized by the dielectric constants $\varepsilon_{a}$ and $\varepsilon_{b}$, respectively. To find the relation between the polarization of the particles and fluctuating field acting in the system under consideration, one needs to take into consideration the free energy of the system, which can be written in the following form [18]

$$
\begin{aligned}
& F=\int_{\Omega_{a}} d \mathbf{R}_{a}\left(U_{\mathrm{int}}^{a}-P_{i}^{a} E_{i}^{e x}-\frac{1}{2} P_{i}^{a} E_{i}^{a}\right)+ \\
& +\int_{\Omega_{a}} d \mathbf{R}_{b}\left(U_{\mathrm{int}}^{b}-P_{i}^{b} E_{i}^{e x}-\frac{1}{2} P_{i}^{b} E_{i}^{b}\right),
\end{aligned}
$$

where $U_{\text {int }}^{a}$ and $U_{\text {int }}^{b}$ are the densities of the internal energy of the dipoles inherent to the particles ' $a$ ' and ' $b$ ', respectively; $P_{i}^{a}$ and $P_{i}^{b}$ are the dipole moments induced in the particles ' $a$ ' and ' $b$ ', respectively; the terms $-P_{i}^{a} E_{i}^{e x}$ and $-P_{i}^{b} E_{i}^{e x}$ mean the energy densities for the dipoles ' $a$ ' and ' $b$ ' under the external electric field according to the system under consideration. When considering the system under a fixed temperature, the entropy term in the free energy can be omitted. The terms $-P_{i}^{a} E_{i}^{a} / 2$ and $-P_{i}^{b} E_{i}^{b} / 2$ describe densities of the interaction energy between the dipoles and local fields. These terms are written in the symmetrized form, and the factor $1 / 2$ is written to take into account pair interactions. We should note that $E_{i}^{a, b}$ is the local field at the dipole, and it differs from the external field acting on the system $E_{i}^{e x}$.

Let us suppose that the external field is lower than intramolecular fields. In this case, the addition to the internal energy of the particle caused by the dipole momentum of this particle will be lower than the energy of the chemical bonds in the material. Then, one can write the internal energies of the particles ' $a$ ' and ' $b$ ' as the expansion in a Taylor series over the dipole momentum $P$ accurate within $P^{4}[19,20]$

$$
\begin{aligned}
& U_{\mathrm{int}}^{(\alpha)}=U_{0}^{(\alpha)}+\frac{1}{\varepsilon_{0}} \frac{1}{2} \lambda_{i j}^{(\alpha)} P_{i}^{(\alpha)} P_{j}^{(\alpha)}+ \\
& +\frac{1}{\varepsilon_{0}} \frac{1}{4} \beta_{i j r s}^{(\alpha)} P_{i}^{(\alpha)} P_{j}^{(\alpha)} P_{r}^{(\alpha)} P_{s}^{(\alpha)},
\end{aligned}
$$

where tensor parameters $\lambda_{i j}^{(\alpha)}$ and $\beta_{i j r s}^{(\alpha)}$ describe the linear and nonlinear polarizabilities of the particles, respectively. The first term in the series $U_{0}^{(\alpha)}$ is the internal energy of the particle (caused by interatomic bonds inside the particle) which does not depend on the dipole momentum $P_{i}^{(\alpha)}$ and can be omitted when the ground state of the system will be found. It should be noted that because of nonpolar particles, one should consider the particles symmetry group which contains the inversion centre (this is the reason why the cubic nonlinear term in Eq. (2) is omitted). To find the energy of interparticle interaction, it is necessary to ascertain the relation between the dipole momentum induced in the particle and external field. To make it, one should write the exact form of the free energy and minimize it over the induced dipole momenta. Then, taking into account that particles under consideration are nonpoint, one can write the free energy of the system in the form

$$
\begin{aligned}
& F=\int_{\Omega_{a}} d \mathbf{R}_{a}\left\{\frac{1}{2} \frac{1}{\varepsilon_{0}} \lambda_{i j}^{a} P_{i}^{a}\left(\mathbf{R}_{a}\right) P_{j}^{a}\left(\mathbf{R}_{a}\right)+\right. \\
& +\frac{1}{4} \frac{1}{\varepsilon_{0}} \beta_{i j r s}^{a} P_{i}^{a}\left(\mathbf{R}_{a}\right) P_{j}^{a}\left(\mathbf{R}_{a}\right) P_{r}^{a}\left(\mathbf{R}_{a}\right) P_{s}^{a}\left(\mathbf{R}_{a}\right)- \\
& \left.+P_{i}^{a}\left(\mathbf{R}_{a}\right) E_{i}^{e x}\left(\mathbf{R}_{a}\right)-\frac{1}{2} P_{i}^{a}\left(\mathbf{R}_{a}\right) E_{i}^{a}\left(P_{i}^{a}, P_{i}^{b}, \mathbf{R}_{a}\right)\right\}+ \\
& +\int_{\Omega_{b}} d \mathbf{R}_{b}\left\{\frac{1}{2} \frac{1}{\varepsilon_{0}} \lambda_{i j}^{b} P_{i}^{b}\left(\mathbf{R}_{b}\right) P_{j}^{b}\left(\mathbf{R}_{b}\right)+\right. \\
& +\frac{1}{4} \frac{1}{\varepsilon_{0}} \beta_{i j r s}^{b} P_{i}^{b}\left(\mathbf{R}_{b}\right) P_{j}^{b}\left(\mathbf{R}_{b}\right) P_{r}^{b}\left(\mathbf{R}_{b}\right) P_{s}^{b}\left(\mathbf{R}_{b}\right)- \\
& \left.+P_{i}^{b}\left(\mathbf{R}_{b}\right) E_{i}^{e x}\left(\mathbf{R}_{b}\right)-\frac{1}{2} P_{i}^{b}\left(\mathbf{R}_{b}\right) E_{i}^{b}\left(P_{i}^{a}, P_{i}^{b}, \mathbf{R}_{b}\right)\right\},
\end{aligned}
$$

where integrations are performed over the volumes of the particles ' $\mathrm{a}$ ' $-\Omega_{a}$, and ' $\mathrm{b}$ ' $-\Omega_{b}$. In this expression, $E_{i}^{e x}\left(\mathbf{R}_{a}\right)$ is the electric field external to the system of polarized particles, and it does not depend on the dipole momenta $P_{i}^{(\alpha)}$. The fields $E_{i}^{a}\left(P_{i}^{a}, P_{i}^{b}, \mathbf{R}_{a}\right)$ and $E_{i}^{b}\left(P_{i}^{a}, P_{i}^{b}, \mathbf{R}_{b}\right)$ mean the local fields at the particles ' $\mathrm{a}$ ' and 'b' (centers of which are situated in the points $\mathbf{R}_{a}$ and $\mathbf{R}_{b}$, respectively) induced by the dipole momenta at the particles ' $b$ ' and ' $a$ '. To find the ground state of the system, one should minimize the energy of the system by the dipole momenta $P_{k}^{a, b}[21,23]$

$$
\left\{\begin{array}{l}
\delta F\left(P_{i}^{a}\left(\mathbf{R}_{a}\right),\left(\left.P_{i}^{b}\left(\mathbf{R}_{b}\right)\right|_{P_{i}^{a}\left(\mathbf{R}_{a}\right)}=0,\right.\right. \\
\delta F\left(P_{i}^{a}\left(\mathbf{R}_{a}\right),\left(\left.P_{i}^{b}\left(\mathbf{R}_{b}\right)\right|_{P_{i}^{b}\left(\mathbf{R}_{b}\right)}=0,\right.\right.
\end{array}\right.
$$

with taking into account the connection between the field in an arbitrary point and the dipole momentum densities at the particles [24]

$$
\begin{aligned}
& E_{j}^{a}\left(\mathbf{R}_{a}\right)=\frac{k_{0}^{2}}{\varepsilon_{0}} \int_{\Omega_{a}} d \mathbf{R}_{a}^{\prime} G_{j i}\left(\mathbf{R}_{a}, \mathbf{R}_{a}^{\prime}\right) P_{i}^{a}\left(\mathbf{R}_{a}^{\prime}\right)+ \\
& +\frac{k_{0}^{2}}{\varepsilon_{0}} \int_{\Omega_{b}} d \mathbf{R}_{b}^{\prime} G_{j i}\left(\mathbf{R}_{a}, \mathbf{R}_{b}^{\prime}\right) P_{i}^{b}\left(\mathbf{R}_{b}^{\prime}\right) .
\end{aligned}
$$


The aim of the present calculations is to express the dipole momenta at the particles via the external field $E_{j}^{e x}(\mathbf{R})$. It is noteworthy that in general case the variation procedure will be very complicated. To simplify the problem without loss of generality, one can suppose that the tensors $\lambda_{i j}^{a, b}, \beta_{i j r s}^{a, b}$ and $G_{i j}\left(\mathbf{R}, \mathbf{R}^{\prime}\right)$ are symmetric. Then, writing Eqs (4) in the explicit form, one obtains

$$
\begin{aligned}
& \left.\delta F\right|_{P_{i}^{a}\left(\mathbf{R}_{a}\right)}=\int_{\Omega_{a}} d \mathbf{R}_{a}\left\{\frac{1}{\varepsilon_{0}} \lambda_{i j}^{a} P_{j}^{a}\left(\mathbf{R}_{a}\right)+\right. \\
& +\frac{1}{\varepsilon_{0}} \beta_{i j r s}^{a} P_{j}^{a}\left(\mathbf{R}_{a}\right) P_{r}^{a}\left(\mathbf{R}_{a}\right) P_{s}^{a}\left(\mathbf{R}_{a}\right)- \\
& -E_{i}^{e x}\left(P_{i}^{a}, P_{i}^{b}, \mathbf{R}_{a}\right)-\frac{k_{0}^{2}}{\varepsilon_{0}} \int_{\Omega_{a}} d \mathbf{R}_{a}^{\prime} G_{j i}\left(\mathbf{R}_{a}^{\prime}, \mathbf{R}_{a}\right) P_{j}^{a}\left(\mathbf{R}_{a}^{\prime}\right)- \\
& \left.-\frac{1}{2} \frac{k_{0}^{2}}{\varepsilon_{0}} \int_{\Omega_{b}} d \mathbf{R}_{b} G_{j i}\left(\mathbf{R}_{b}, \mathbf{R}_{a}\right) P_{j}^{b}\left(\mathbf{R}_{b}\right)\right\} \delta P_{i}^{a}\left(\mathbf{R}_{a}\right)=0, \\
& \left.\delta F\right|_{P_{i}^{b}\left(\mathbf{R}_{b}\right)}=\int_{\Omega_{b}} d \mathbf{R}_{b}\left\{\frac{1}{\varepsilon_{0}} \lambda_{i j}^{b} P_{j}^{b}\left(\mathbf{R}_{b}\right)+\right. \\
& +\frac{1}{\varepsilon_{0}} \beta_{i j r s}^{b} P_{j}^{b}\left(\mathbf{R}_{b}\right) P_{r}^{b}\left(\mathbf{R}_{b}\right) P_{s}^{b}\left(\mathbf{R}_{b}\right)- \\
& -E_{i}^{e x}\left(P_{i}^{a}, P_{i}^{b}, \mathbf{R}_{b}\right)-\frac{k_{0}^{2}}{\varepsilon_{0}} \int_{\Omega_{a}} d \mathbf{R}_{b}^{\prime} G_{j i}\left(\mathbf{R}_{b}^{\prime}, \mathbf{R}_{b}\right) P_{j}^{b}\left(\mathbf{R}_{b}^{\prime}\right)- \\
& \left.-\frac{1}{2} \frac{k_{0}^{2}}{\varepsilon_{0}} \int_{\Omega_{b}} d \mathbf{R}_{b} G_{j i}\left(\mathbf{R}_{b}, \mathbf{R}_{a}\right) P_{j}^{a}\left(\mathbf{R}_{a}\right)\right\} \delta P_{i}^{b}\left(\mathbf{R}_{b}\right)=0 .
\end{aligned}
$$

Performed minimization gives the system of equations that enables us to find the following relations between polarizations at the particles $-P_{i}^{a, b}\left(\mathbf{R}_{a, b}\right)$ and the external field $E_{j}^{e x}(\mathbf{R})$

$$
\begin{aligned}
& \frac{1}{\varepsilon_{0}} \lambda_{i j}^{a} P_{j}^{a}\left(\mathbf{R}_{a}\right)+\frac{1}{\varepsilon_{0}} \beta_{i j r s}^{a} P_{j}^{a}\left(\mathbf{R}_{a}\right) P_{r}^{a}\left(\mathbf{R}_{a}\right) P_{s}^{a}\left(\mathbf{R}_{a}\right)- \\
& -E_{i}^{e x}\left(\mathbf{R}_{a}\right)-\frac{k_{0}^{2}}{\varepsilon_{0}} \int_{\Omega_{a}} d \mathbf{R}_{a}^{\prime} G_{i j}\left(\mathbf{R}_{a}, \mathbf{R}_{a}^{\prime}\right) P_{j}^{a}\left(\mathbf{R}_{a}^{\prime}\right)- \\
& -\frac{1}{2} \frac{k_{0}^{2}}{\varepsilon_{0}} \int_{\Omega_{b}} d \mathbf{R}_{b}^{\prime} G_{i j}\left(\mathbf{R}_{a}, \mathbf{R}_{b}^{\prime}\right) P_{j}^{b}\left(\mathbf{R}_{b}^{\prime}\right)=0, \\
& \frac{1}{\varepsilon_{0}} \lambda_{i j}^{b} P_{j}^{b}\left(\mathbf{R}_{b}\right)+\frac{1}{\varepsilon_{0}} \beta_{i j r s}^{b} P_{j}^{b}\left(\mathbf{R}_{b}\right) P_{r}^{b}\left(\mathbf{R}_{b}\right) P_{s}^{b}\left(\mathbf{R}_{b}\right)- \\
& -E_{i}^{e x}\left(\mathbf{R}_{b}\right)-\frac{k_{0}^{2}}{\varepsilon_{0}} \int_{\Omega_{a}} d \mathbf{R}_{b}^{\prime} G_{i j}\left(\mathbf{R}_{b}, \mathbf{R}_{b}^{\prime}\right) P_{j}^{b}\left(\mathbf{R}_{b}^{\prime}\right)- \\
& -\frac{1}{2} \frac{k_{0}^{2}}{\varepsilon_{0}} \int_{\Omega_{b}} d \mathbf{R}_{a}^{\prime} G_{i j}\left(\mathbf{R}_{b}, \mathbf{R}_{a}^{\prime}\right) P_{j}^{b}\left(\mathbf{R}_{a}^{\prime}\right)=0 .
\end{aligned}
$$

(C) 2012, V. Lashkaryov Institute of Semiconductor Physics, National Academy of Sciences of Ukraine
Eqs. (8) and (9) are nonlinear integral equations and, in general, cannot be strictly solved analytically. Then, the solutions can be found by the method of successive approximations [25-27] with the assumption that free particles have not dipole momentum and external field is long-range. It means that one should find the solutions in the form

$$
\begin{aligned}
& P_{i}^{a, b}\left(\mathbf{R}_{a, b}\right)=P_{i}^{a, b(0)}\left(\mathbf{R}_{a, b}\right)+P_{i}^{a, b(1)}\left(\mathbf{R}_{a, b}\right)+ \\
& +P_{i}^{a, b(2)}\left(\mathbf{R}_{a, b}\right)+\ldots
\end{aligned}
$$

Zero-order approximation can be found in the assumption that the particles are linear and noninteracting. Then, one can suppose that relation between the dipole momentum at the particle and the external (relatively to the particle) field is linear, namely:

$$
P_{i}^{a, b(0)}\left(\mathbf{R}_{a, b}\right)=\varepsilon_{0} X_{i j}^{(0) a, b}\left(\mathbf{R}_{a, b}\right) E_{j}^{e x}\left(\mathbf{R}_{a, b}\right) .
$$

Then, one obtains from Eq.(8)

$$
\begin{aligned}
& \lambda_{i j}^{a} X_{j l}^{a}\left(\mathbf{R}_{a}\right) E_{l}^{e x}\left(\mathbf{R}_{a}\right)=E_{i}^{e x}\left(\mathbf{R}_{a}\right)+ \\
& +k_{0}^{2} \int_{\Omega_{a}} d \mathbf{R}_{a}^{\prime} G_{i j}\left(\mathbf{R}_{a}, \mathbf{R}_{a}^{\prime}\right) X_{j l}^{a}\left(\mathbf{R}_{a}^{\prime}\right) E_{l}^{e x}\left(\mathbf{R}_{a}^{\prime}\right) .
\end{aligned}
$$

To find the solution of Eq. (12), let us integrate both (left and right) parts of the equation over the volume of the particle ' $a$ '

$$
\begin{aligned}
& \int_{\Omega_{a}} d \mathbf{R}_{a}\left\{\lambda_{i j}^{a} X_{j l}^{a}\left(\mathbf{R}_{a}\right)-\delta_{i l}-\right. \\
& \left.-k_{0}^{2} \int_{\Omega_{a}} d \mathbf{R}_{a}^{\prime} G_{i j}\left(\mathbf{R}_{a}^{\prime}, \mathbf{R}_{a}\right) X_{j l}^{a}\left(\mathbf{R}_{a}\right)\right\} E_{l}^{e x}\left(\mathbf{R}_{a}\right) .
\end{aligned}
$$

Taking into account that external field is, generally speaking, arbitrary, and integration is over any particle, and writing the external field as

$$
E_{l}^{e x}\left(\mathbf{R}_{a}\right)=\sum_{\mathbf{k}} E_{l}^{e x}(\mathbf{k}) e^{i \mathbf{k} \mathbf{R}_{a}}
$$

one can obtain

$$
\begin{aligned}
& \sum_{\mathbf{k}} \int_{\Omega_{a}} d \mathbf{R}_{a}\left\{\lambda_{i j}^{a} X_{j l}^{a}\left(\mathbf{R}_{a}\right)-\delta_{i l}-\right. \\
& \left.-k_{0}^{2} \int_{\Omega_{a}} d \mathbf{R}_{a}^{\prime} G_{i j}\left(\mathbf{R}_{a}^{\prime}, \mathbf{R}_{a}\right) X_{j l}^{a}\left(\mathbf{R}_{a}\right)\right\} e^{i \mathbf{k} \mathbf{R}_{a}} E_{l}^{e x}(\mathbf{k}) .
\end{aligned}
$$

Exponents $\exp \left(i \mathbf{k} \mathbf{R}_{\mathrm{a}}\right)$ are the orthonormal basis. Then, to satisfy Eq. (15), one must put to zero the expression in the brackets. As a result, one obtains

$$
X_{j l}^{a}\left(\mathbf{R}_{a}\right)=\delta_{i l}\left[\lambda_{i j}^{a}-k_{0}^{2} \int_{\Omega_{a}} d \mathbf{R}_{a}^{\prime} G_{i j}\left(\mathbf{R}_{a}^{\prime}, \mathbf{R}_{a}\right)\right]^{-1} .
$$

This expression has the same form as the expression of effective susceptibility obtained in [28]. The effective susceptibility describes the relation between the local polarization inside the particle and 
external field. One should note that the coordinate $\mathbf{R}_{a}$ in Eq. (16) is a coordinate inside the particle ' $a$ '. As it follows from the above speculations, the effective susceptibility relates the local polarization inside the nanoparticle with an external field acting to the particle

$$
P_{j}\left(\mathbf{R}_{a}\right)=X_{j l}^{a}\left(\mathbf{R}_{a}\right) E_{l}^{e x}\left(\mathbf{R}_{a}\right) .
$$

In this case, the expression [Eq. (17)] is very similar to a constitutive equation. Then, one can claim that unlike to the constitutive equation that describes the characteristic of a material, Eq. (17) describes the characteristic of an object. As it is known from [29], the term $k_{0}^{2} \int_{\Omega_{a}} d \mathbf{R}_{a}^{\prime} G_{i j}\left(\mathbf{R}_{a}^{\prime}, \mathbf{R}_{a}\right)$ in Eq. (16) plays a role of self-energy part and describes the interactions inside the particle. In the case when interparticle interactions vanish, we obtain the susceptibility of the material from which the particle is made. Then, it is easy to find the physical treatment of the coefficient $\lambda_{j l}^{a}$ in the series [Eq. (2)] $\lambda_{j l}^{a}=\left(\alpha_{j l}^{a}\right)^{-1}$. Analogously to Eq. (15), one can obtain the effective susceptibility for the particle ' $b$ '.

To obtain the first iteration, one should substitute in Eq. (8) $P_{i}(\mathbf{R})=P_{i}^{(0)}(\mathbf{R})+P_{i}^{(1)}(\mathbf{R})$. Then, one can write the equation of the first order iteration

$$
\begin{aligned}
& P_{j}^{(1)}\left(\mathbf{R}_{a}\right)=-\alpha_{j i}^{a} \beta_{i l r s}^{a} P_{l}^{a(0)}\left(\mathbf{R}_{a}\right) P_{r}^{a(0)}\left(\mathbf{R}_{a}\right) P_{s}^{a(0)}\left(\mathbf{R}_{a}\right)+ \\
& +\frac{k_{0}^{2}}{2} \int_{\Omega_{b}} d \mathbf{R}_{b}^{\prime} \alpha_{j i}^{a} G_{i l}\left(\mathbf{R}_{a}, \mathbf{R}_{b}^{\prime}\right) P_{l}^{b(0)}\left(\mathbf{R}_{b}^{\prime}\right) .
\end{aligned}
$$

Substitution of Eq. (11) into this equation gives $P_{j}^{a(1)}\left(\mathbf{R}_{a}\right)=\varepsilon_{0} N_{j k l m}^{(1) a} E_{k}^{e x}\left(\mathbf{R}_{a}\right) E_{l}^{e x}\left(\mathbf{R}_{a}\right) E_{m}^{e x}\left(\mathbf{R}_{a}\right)+$ $+\varepsilon_{0} X_{j l}^{(1) b} E_{l}^{e x}\left(\mathbf{R}_{b}\right)$,

where

$N_{j k l m}^{(1) a}=-\varepsilon_{0}^{2} \alpha_{j i}^{a} \beta_{i l r s}^{a} X_{l k}^{a}\left(\mathbf{R}_{a}\right) X_{r m}^{a}\left(\mathbf{R}_{a}\right) X_{s l}^{a}\left(\mathbf{R}_{a}\right)$,

$X_{j l}^{(1) b}=\frac{k_{0}^{2}}{2} \int_{\Omega_{b}} d \mathbf{R}_{b}^{\prime} \alpha_{j i}^{a} G_{i l}\left(\mathbf{R}_{a}, \mathbf{R}_{b}^{\prime}\right) X_{l k}^{b}\left(\mathbf{R}_{b}^{\prime}\right)$.

Obviously the same expression can be obtained for the particle ' $b$ '. Then, one can derive the relation connecting the polarization inside the particle characterized both by linear and nonlinear polarizabilities in the first order iteration (accurate within the linear term of nonlinearity tensor $\beta_{i l r s}^{a}$, $\alpha=a, b)$

$P_{i}^{a, b}\left(\mathbf{R}_{a, b}\right)=\varepsilon_{0} X_{i j}^{(0) a, b}\left(\mathbf{R}_{a, b}\right) E_{j}^{e x}\left(\mathbf{R}_{a, b}\right)+$

$+\varepsilon_{0} N_{j k l m}^{(1) a} E_{k}^{e x}\left(\mathbf{R}_{a}\right) E_{l}^{e x}\left(\mathbf{R}_{a}\right) E_{m}^{e x}\left(\mathbf{R}_{a}\right)+$

$+\varepsilon_{0} X_{j l}^{(1) b} E_{l}^{e x}\left(\mathbf{R}_{b}\right)$.

This expression can be used to establish the interparticle interaction potential, which will be developed in the next chapter.

\subsection{The interaction potential}

The part of the energy of the system depending on the distance between the particles has a sense of interaction potential (binding energy) and can be defined as

$$
\begin{aligned}
& U(d)=-\int_{\Omega_{a}} d \mathbf{R}_{a}\left(P_{i}^{a} E_{i}^{e x}+\frac{1}{2} P_{i}^{a} E_{i}^{a}\right)- \\
& -\int_{\Omega_{b}} d \mathbf{R}_{b}\left(P_{i}^{b} E_{i}^{e x}+\frac{1}{2} P_{i}^{b} E_{i}^{b}\right)-\delta U,
\end{aligned}
$$

where $d$ is the distance between the particles, and the last term

$$
\begin{gathered}
\delta U=-\int_{\Omega_{a}} d \mathbf{R}_{a}\left(P_{i}^{a} E_{i}^{e x}+\frac{1}{2} P_{i}^{a} E_{i}^{a}\right)- \\
-\left.\int_{\Omega_{b}} d \mathbf{R}_{b}\left(P_{i}^{b} E_{i}^{e x}+\frac{1}{2} P_{i}^{b} E_{i}^{b}\right)\right|_{d \rightarrow \infty},
\end{gathered}
$$

is the energy of the system when the particles are pulled apart one from another to infinity. As it can be easily seen from Eq. (22), to find the function of interparticle interaction as a function of the distance $d$, one has to know the explicit form of the self-consistent local field of the system. Then, to calculate the interaction potential, one should put Eqs (5) and (21) into Eq. (22). As a result, one obtains the explicit expression for the interaction potential, depending on external field acting to the particles composing the system under consideration

$$
\begin{aligned}
& U(d)=\sum_{\alpha=a, b \beta=a, b} \sum_{k l} C^{(\alpha, \beta)}(d) E_{k}^{e x}\left(\mathbf{R}_{\alpha}\right) E_{l}^{e x}\left(\mathbf{R}_{\beta}\right)+ \\
& +\sum_{\alpha=a, b \beta=a, b} \sum_{l m p q} D_{l(\alpha, \beta)}(d) E_{l}^{e x}\left(\mathbf{R}_{\alpha}\right) E_{m}^{e x}\left(\mathbf{R}_{\beta}\right) E_{p}^{e x}\left(\mathbf{R}_{\alpha}\right) E_{q}^{e x}\left(\mathbf{R}_{\beta}\right) \text {, }
\end{aligned}
$$

where coefficients $C_{k l}^{(\alpha, \beta)}(d)$ and $D_{l m p q}^{(\alpha, \beta)}$ are expressed via linear and nonlinear polarizabilities of the particles and the electrodynamic Green function of the medium where the nanoparticles are placed. One should note that the external field was taken outside integrals because of their long-range nature.

In the case when the external field controlled by experimentalist is absent, the interaction potential can be differed from zero because of the field of vacuum fluctuations $E_{j}^{(0)}\left(\mathbf{R}_{\alpha}\right)$. With account of rather small linear dimensions of the particles under consideration, one takes into account only long-range components of fluctuating field. Then, one can use Eq. (24) where the statistical averaging is made [30]. As a result, one obtains

$$
\begin{aligned}
& U(d)=\sum_{\alpha=a, b \beta=a, b} \sum_{k l} C^{(\alpha, \beta)}(d)\left\langle E_{k}^{(0)}\left(\mathbf{R}_{\alpha}\right) E_{l}^{(0)}\left(\mathbf{R}_{\beta}\right)\right\rangle+ \\
& +\sum_{\alpha=a, b \beta=a, b} \sum_{l m p q} D^{(\alpha, \beta)}(d) E_{l}^{e x}\left(\mathbf{R}_{\alpha}\right) E_{m}^{e x}\left(\mathbf{R}_{\beta}\right) E_{p}^{e x}\left(\mathbf{R}_{\alpha}\right) E_{q}^{e x}\left(\mathbf{R}_{\beta}\right),
\end{aligned}
$$


The terms $C_{k l}^{(\alpha, \beta)}(d)$ and $D_{l m p q}^{(\alpha, \beta)}$ are very lengthy and will not be written here in an explicit form. Because of 2-rank correlator spectral densities can be easily calculated, one should represent correlators $\left\langle E_{i}^{0}\left(\mathbf{R}_{a, b}\right) E_{j}^{0}\left(\mathbf{R}_{a, b}\right) E_{k}^{0}\left(\mathbf{R}_{a, b}\right) E_{l}^{0}\left(\mathbf{R}_{a, b}\right)\right\rangle_{\omega}$ via 2-rank correlators, according to the rule analogous to Wick's theorem [31]

$\left\langle E_{i}^{0}\left(\mathbf{R}_{a, b}\right) E_{j}^{0}\left(\mathbf{R}_{a, b}\right) E_{k}^{0}\left(\mathbf{R}_{a, b}\right) E_{l}^{0}\left(\mathbf{R}_{a, b}\right)\right\rangle_{\omega}=$

$\sum_{a, b}\left\langle E_{i}^{0}\left(\mathbf{R}_{a, b}\right) E_{j}^{0}\left(\mathbf{R}_{a, b}\right) E_{k}^{0}\left(\mathbf{R}_{a, b}\right) E_{l}^{0}\left(\mathbf{R}_{a, b}\right)\right\rangle_{\omega}$

with different commutation of indexes $i, j, k, l$ and $a, b$.

Then the interaction potential can be expressed as

$U(d)=\int_{-\infty}^{\infty} \frac{d \omega}{2 \pi} \frac{\hbar}{1-\exp (-\hbar \omega / k T)} \times$

$\times\left\{\sum_{\alpha=a, b \beta=a, b} \sum_{k l} C^{(\alpha, \beta)}(d)\left\langle E_{k}^{0}\left(\mathbf{R}_{\alpha}\right) E_{l}^{0}\left(\mathbf{R}_{\beta}\right)\right\rangle_{\omega}+\right.$

$\left.+\sum_{\alpha=a, b \beta=a, b} \sum_{l m p q}(d)\left\langle E_{l}^{(0)}\left(\mathbf{R}_{\alpha}\right) E_{m}^{(0)}\left(\mathbf{R}_{\beta}\right)\right\rangle_{\omega}\left\langle E_{p}^{(0)}\left(\mathbf{R}_{\alpha}\right) E_{q}^{(0)}\left(\mathbf{R}_{\beta}\right)\right\rangle_{\omega}\right\}$.

The correlator $\left\langle E_{i}^{(0)}\left(\mathbf{R}_{\alpha}\right) E_{j}^{(0)}\left(\mathbf{R}_{\beta}\right)\right\rangle_{\omega}$, in its turn, can be expressed via photon propagator of medium in which the particles are situated using the Callen-Welton theorem [32]. As it was shown in [30], one can express the spectral density of the correlator as

$$
\left\langle E_{i}^{(0)}\left(\mathbf{R}_{\alpha}\right) E_{j}^{(0)}\left(\mathbf{R}_{\beta}\right)\right\rangle_{\omega}=\frac{\omega^{2}}{c^{2}} \operatorname{Im} D_{i j}^{0}\left(\omega, \mathbf{R}_{b}-\mathbf{R}_{a}\right) \operatorname{sign} \omega,
$$

where $D_{i j}^{0}\left(\omega, \mathbf{R}_{b}-\mathbf{R}_{a}\right)$ is the photon propagator, $\omega-$ frequency, $c-$ speed of light, sign $\omega=-1$, if $\omega<0$, $\operatorname{sign} \omega=1$, if $\omega>0$, sign $\omega=0$, if $\omega=0$. In the near-field approximation (when the retardation processes are neglected). In the case when one should calculate the correlators at the same points $\left\langle E_{i}^{(0)}\left(\mathbf{R}_{\alpha}\right) E_{j}^{(0)}\left(\mathbf{R}_{\alpha}\right)\right\rangle_{\omega}$, one can use [30]

$$
\left\langle E_{i}^{(0)}\left(\mathbf{R}_{a}\right) E_{j}^{(0)}\left(\mathbf{R}_{a}\right)\right\rangle_{\omega}=\frac{2}{3} \hbar \frac{\omega^{3}}{c^{3}} \delta_{i j} \text { sign } \omega .
$$

As a result, one can write the explicit form of interparticle potential in the near-field approximation

$U(d)=\frac{A_{a, b}}{d^{12}}-\frac{B_{a, b}}{d^{6}}$,

with coefficients $A_{a, b}$ and $B_{a, b}$ depending on the type (dimension, shape and material from which the particles are fabricated). These coefficients were calculated with Eqs (27) to (29), but because of the explicit form of these coefficients are very lengthy we did not write these equations here. As it is seen from Eq. (30), the interaction potential is similar to the Lennard-Jones potential and has both repulsive and attractive terms.

Using the expressions given above, one can evaluate the potential of interparticle interaction for the particles of various shapes and dimensions.

\section{Numerical calculations, results and discussion}

To illustrate the above obtained interaction potential between two nonpoint particles, we have presented here some results of numerical calculations for the interaction potential. To simplify the calculations, we have supposed that the long-range fluctuation fields mainly contribute to formation of the potential. Due to accounting for only rather small particles (not larger than $100 \mathrm{~nm}$ ), we used the near-field approximation, in the frame of which the electrodynamic Green function [34] was used

$$
G_{i l}\left(\mathbf{R}, \mathbf{R}^{\prime}\right)=\frac{1}{4 \pi k_{0}^{2} \widetilde{R}^{3}}\left[\delta_{i j}-\frac{3 \widetilde{R}_{i} \widetilde{R}_{j}}{\widetilde{R}^{2}}\right], \widetilde{R}=\mathbf{R}-\mathbf{R}^{\prime} .
$$

The coordinates of centers for the particles ' $a$ ' and 'b' are taken as $\mathbf{R}_{a}^{0}=\{0,0, d / 2\}, \mathbf{R}_{b}^{0}=\{0,0,-d / 2\}$, respectively. Then, one can see that the main contribution is given by the correlators $\left\langle E_{z}^{(0)}(\mathbf{R}) E_{z}^{(0)}\left(\mathbf{R}^{\prime}\right)\right\rangle_{\omega}$. Taking into account these circumstances, we calculated the interaction potentials between two nanoparticles made from different materials (characterized by dielectric constant $\varepsilon$ ), and characterized by different shapes and dimensions. Results of these calculations are shown in Figs 2 and 3. The interaction potentials describing interaction between two particles larger of which is a sphere with the radius $50 \mathrm{~nm}$, or smaller particles, each of which has the volume $V_{S}=4186 \mathrm{~nm}^{3}$ (corresponds to spherical particle of the radii $10 \mathrm{~nm}$ ) are shown in Fig. 2. The first characteristic feature of the potential is formation of minimum at the distance close to linear dimensions of the smaller particle. The second characteristic feature of the potential is its dependence on the shape of particles. The dependence of interparticle potential on dielectric constant of smaller particle, when the both particles are spherical, is shown in Fig. 3. The radii of the particles are 50 and $10 \mathrm{~nm}$. The dielectric constant of the larger particle equals to 2 . One can see that the potential strongly depends on the dielectric constant of material from which the particles are fabricated. Nevertheless, one can see that in a rather wide range of values of dielectric constants the potential can have a minimum that takes place at distances close to linear dimension of the smaller particle. Of course, it seems reasonable to perform similar calculations of the interaction potentials for different shapes, dimensions and dielectric constants of the particles. The results of the calculations show that the interaction potential can have both attractive and repulsive terms and strongly depends on 
- shape and dimension of the particles; fabricated;

materials from which the particles are

- mutual orientation of the particles.

All these properties allow us to suppose that the proposed mechanism of interparticle interaction can be used in biology and medicine for different purposes.

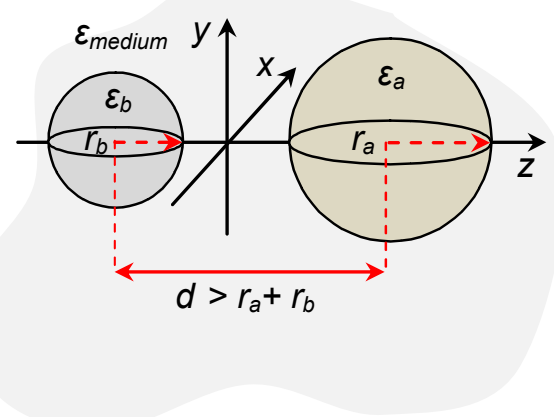

Fig. 1. (Color online) Sketch of the system geometry.

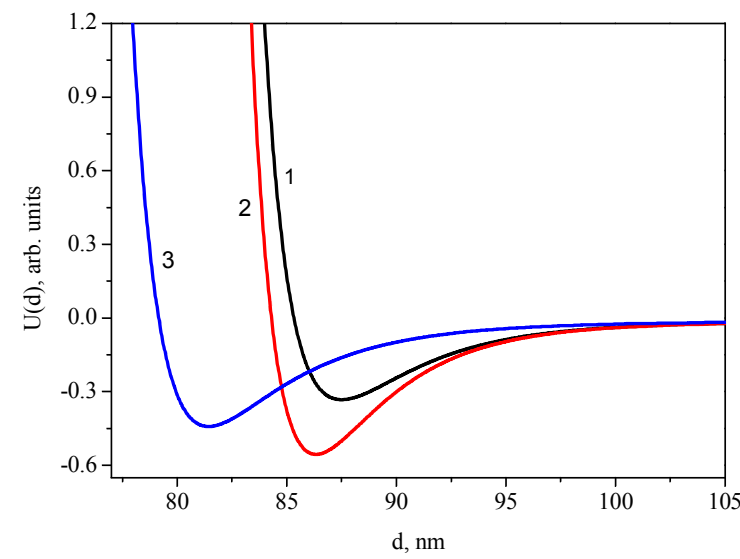

Fig. 2. Interaction potential for the particles of different shapes. Curve 1 corresponds to two spherical particles, curve 2 prolate ellipsoidal smaller particle, and curve 3 - oblate smaller particle.

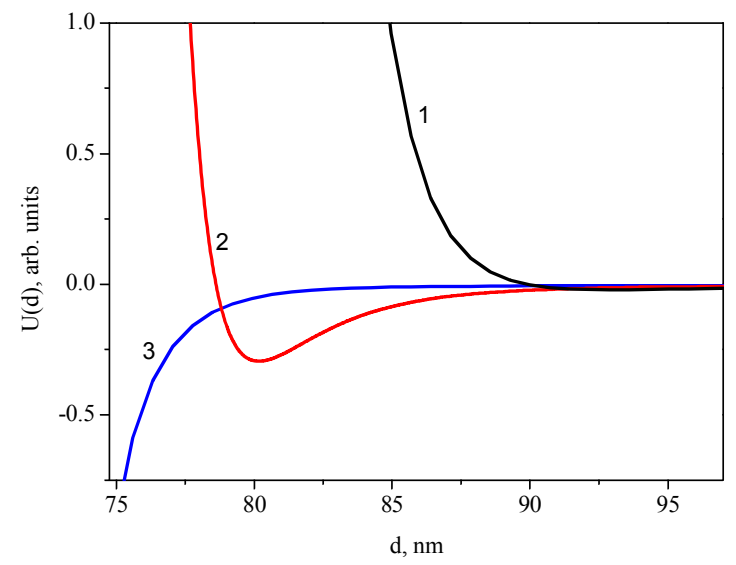

Fig. 3. The dependence of interparticle potential of two spherical particles on dielectric constant of material from which the smaller particle is made. Curve 1 corresponds to $\varepsilon_{s}=$ 10 , curve $2-\varepsilon_{s}=5$, curve $3-\varepsilon_{s}=2$.

\section{Implementation to biology and medicine}

The results obtained in the previous chapter enable to think about the possibility of influence by nanoparticles on living objects - living cells, bacteria and viruses. It is pertinent to consider at least two ways of this influence. First of them is the ability to create a stable formation consisting of living objects and nanoparticles. Second way of the influence lies in the ability of nanoparticle to induce rather strong electric field at the living object due to the so-called local-field enhancement $[33,34]$.

These two aspects of interaction between the living object and nanoparticle are most brightly pronounced in nanoparticle antivirus activities. Indeed, there are a lot of the communications in the scientific journals in which the ability of different types of nanoparticles to suppress the virus infection activity $[11,35,36]$ is reported. In the previous works [11], authors suggested that formation of stabile configurations of 'virus-nanoparticle' can be considered as an universal reason of antiviral activity of the prepared nanoparticles. Indeed, it is clear that there are two definitely different mechanisms of the viral activity inhibition. The inhibition of viral activity consists of two kinds of processes:

I. The first set of the mechanisms is related with abatement of the possibility for virus penetration inside the living cell.

- The penetration of the viruses through the membrane of the cell depends on the geometry of the viral particle. Due to the stability of the complex 'virusnanoparticle' the geometry of the system differs from that of a single virus, which does not allow an efficient interaction between the active centers at the viral capsid and the living cell membrane.

- As it was noted above, the local field action on the receptors at the viral capsid can lead to modification of molecular groups on the receptors including their destruction. As a result, the virus loses its ability to penetrate through the membrane inside the living cell.

- In the case when viruses infected the cell and the processes of viral reproduction start, the viruses of a new generation leaving the cell interact with the nanoparticles and can lose its infection activities via the mechanisms mentioned above.

II. The next mechanism related with penetration of the viruses and nanoparticles inside the living cell.

- In the case when the system 'virus-nanoparticle' penetrates inside the cell, presence of the nanoparticle with its local field action can block the process of virion formation.

- In the case when inside the living cell the single nanoparticles penetrate, the action of the nanoparticle due to the local-field effects can block the virus reproduction by destroying the viral DNA or RNA structure.

Carefully analyzing all the circumstances described above, one can conclude that with the exception of geometrical factors of nanoparticle influence on the virus infection ability, all other terms 
somehow or other are related with the local-field enhancement effect. Indeed, the presence of rather strong local field in molecular structures of virus active centres or intracellular organelles leads to appearance of a term proportional to $-\mathbf{p} \mathbf{E}^{\text {loc }}$ in Hamiltonian describing formation of biospecific bonds. Moreover, the influence on the active centres via the strong local field leads to that the intramolecular bonds of active centres will be modified. This can lead to arising some other properties of the active centres. As a result, the active centres at the virus surface cannot play the role of 'key' to virus penetration into the cell.

On the other side, as both the dipole momentum of intramolecular bonds and local field are caused by the field of vacuum fluctuations. It means that the perturbation term in Hamiltonian should be averaged. As a result, the influence of local-field will appear via correlators of fluctuating field similar to Eqs (28) and (29).

\section{Conclusions}

In this work, the mechanism of interactions between two nanoparticles based on the local-field induced dipole momentum interaction with taking into account nonlinear polarizabilities of the particles is proposed. It was assumed that the dipole momentum induced at the particles can have a fluctuating nature. From this point of view, the proposed interaction is similar to van der Waals interaction (or Casimir effect [37]). Unlike the van der Waals interaction, the proposed mechanism of interparticle interaction leads to formation of both repulsive and attractive terms having the minimum at the distance between particles close to their linear dimensions. The profile and value of the potential depend both on dimensions and shapes of particles as well as their mutual orientations. Implementation of considered interaction between the nanoparticles to biology and medicine has been discussed.

\section{References}

1. B A Joyce, Molecular beam epitaxy // Repts. Progr. Phys. 48(12), p. 1637-1698 (1985).

2. Chang Yang, Wei Chen, Brian Quang Bui, and Guangya Xiang, Recent progress on the liposomes loaded with quantum dots // Rev. Nanosci. Nanotechnol. 1(4), p. 257-270 (2012).

3. Bharat Bhushan, Springer Handbook of Nanotechnology. Springer, 2007.

4. Edward L. Wolf, Nanophysics and Nanotechnology. John Wiley \& Sons, 2006.

5. G.A. Ozin, A.C. Arsenault, L. Cademartiri, Nanochemistry: A Chemical Approach to Nanomaterials. Royal Society of Chemistry Publishing, 2009.

6. Tuan Vo-Dinh, Nanotechnology in Biology and Medicine: Methods, Devices, and Applications. Taylor \& Francis Group, 2007.
7. Rahul Shetty, Nanotechnology: the Future in Medicine. Mesocore Technologies Inc., Quebec, Canada, 2006.

8. X. Huang, P.K. Jain, I.H. El-Sayed, M.A. El-Sayed, Plasmonic photothermal therapy (PPTT) using gold nanoparticles // Lasers Med. Sci. 23, p. 217-228 (2008).

9. B.N. Khlebtsov, E.V. Panfilova, G.S. Terentyuk, I.L. Maksimova, A.V. Ivanov, N.G. Khebtsov, Plasmonic nanopowders for photothermal therapy of tumors // Langmuir, 28(24), p. 8994-9002 (2012).

10. D. Pissuwan, S.M. Valenzuela, and M.B. Cortie, Prospects for gold nanorod particles in diagnostic and therapeutic applications // Biotechnol. and Genetic Eng. Rev. 25, p. 93-112 (2008).

11. V. Lozovski, V. Lysenko, V. Piatnytsia, O. Scherbakov, N. Zholobak, M. Spivak, Physical point of view for antiviral effect caused by the interaction between the viruses and nanoparticles // J. Bionanosci. 6(2), p. 109-112 (2012).

12. L. Zhang, F.X. Gu, J.M. Chan, A.Z. Wang, R.S. Langer and O.C. Farokhzad, Nanoparticles in medicine: Therapeutic applications and developments // Clinical Pharmacology\& Therapeutics, 83(5), p. 761-769 (2008).

13. W.H. De Jong and P.J.A. Borm, Drug delivery and nanoparticles: Applications and hazards // Intern. J. Nanomedicine 3(2), p. 133-149 (2008).

14. A.Z. Wang, R. Langer, and O.C. Farokhzad, Nanoparticle delivery of cancer drugs // Annu. Rev. of Medicine, 63, p. 185-198 (2012).

15. A. Dmitriev, Integrated Analytical Systems: Nanoplasmonic Sensors. Springer, 2012.

16. A. El-Ansary, L.M. Faddah, Nanoparticles as biochemical sensors // Nanotechnology, Science and Applications, 3, p. 65-76 (2010).

17. V. Lozovski, V. Piatnytsia, The potential of the interaction between of two nonpoint nano-particles // J. Computat. Theor. Nanosci. 10 (2013) to be published.

18. L.D. Landau \& E.M. Lifshitz, Electrodynamics of Continuous Media (Vol. 8, A Course of Theoretical Physics). Pergamon Press, 1960.

19. H. Kleinert, Gauge Fields in Condensed Matter, I. World Scientific, Singapore, 1989.

20. V.Z. Lozovski and B.I. Khudik, The new mechanism of physical adsorption on solid surface. I. Adsorption of nonpolar molecules // phys. status solidi (b), 158, p. $511-519$ (1990).

21. E.V. Chenskii and V.V. Tarasenko, Theory of phase transitions into inhomogeneous states in organic ferroelectrics in an external electric field // Zhurnal Experiment. Teor. Fiziki, 83, p. 1089-1099 (1982), in Russian.

22. D.R.S. Talbot, J.R. Wilusima, Variational principles for inhomogeneous nonlinear media // IMA J. Appl. Math. 35(1), p. 39-54 (1985).

23. L. Yang, K. Dayal, A completely iterative method for the infinite domain electrostatic problem with 
nonlinear dielectric media // J. Comput. Phys. 230(21), p. 7821-7829 (2011).

24. O. Keller, Local fields in the electrodynamics of mesoscopic media // Phys. Repts. 268, p. 85-262 (1996).

25. D.K. Faddeev, V.N. Faddeeva, Computational Methods of Linear Algebra. Freeman, 1963.

26. V.I. Krylov, V.V. Bobkov, P.I. Monastyrnyi, Numerical Methods, 1-2. Nauka, Moscow, 1977 (in Russian).

27. L. Collatz, Funktionalanalysis und Numerische Mathematik. Springer, 1964.

28. V. Lozovski, The effective susceptibility concept in the electrodynamics of nano-systems // J. Computat. Theor. Nanosci. 7(10), p. 2077-2093 (2010).

29. A.A. Abrikosov, L.P. Gor'kov, I.E. Dzyaloshinskii, Quantum Field Theoretical Methods in Statistical Physics, 2ed. Pergamon, 1965.

30. Yu.S. Barash and V.L. Ginzburg, Some problems in the theory of Van der Waals forces // Uspekhi Fiz. Nauk, 27(7), p. 467-493 (1984), in Russian.

31. W.H. Dickhoff, D. Van Neck, Many-Body Theory Exposed! Propagator Description of Quantum Mechanics Many-Body Systems. World Scientific, 2005.
32. H.B. Callen, T.A. Welton, Irrevesibility and generalized noise // Phys. Rev. 83(1) p. 34-40 (1951).

33. K. Tanabe, Field enhancement around metal nanoparticles and nanoshells: A systematic investigation // J. Phys. Chem. C, 112(40), p. 15721-15728 (2008).

34. A.R. Guerrero, Yun Zhang, R.F. Aroca, Experimental confirmation of local field enhancement determining far-field measurements with shell-isolated silver nanoparticles // Small 8(19), p. 2964-2967 (2012).

35. H.H. Lara, N.V. Ayala-Nuñez, L. Ixtepan-Turrent and C. Rodriguez-Padilla, Mode of antiviral action of silver nanoparticles against HIV-1 // J. Nanobiotechnology, 8(1), p. 10 (2010).

36. N.A. Mazurkova, Yu.E. Spitsyna, N.V. Shikina, Z.R. Ismagilov, S.N. Zagrebel'nyi, and E.I. Ryabchikova, Interaction of titanium dioxide nanoparticles with influenza virus // Nanotechnologies in Russia, 5(5-6), p. 417-420 (2010)

37. R. Jaffe, Casimir effect and the quantum vacuum // Phys. Rev. D, 72(2), 021301 (2005). 